\title{
Salm, Steven J. \& Falola, Toyin (eds.). - African Urban Spaces in Historical Perspective
}

Rochester-Woodbridge, University of Rochester Press, 2005, XL+397 p., index.

\section{Odile Goerg}

\section{OpenEdition}

\section{Journals}

\section{Édition électronique}

URL : http://journals.openedition.org/etudesafricaines/6858

DOI : $10.4000 /$ etudesafricaines.6858

ISSN : $1777-5353$

Éditeur

Éditions de l'EHESS

\section{Édition imprimée}

Date de publication : 7 mars 2007

Pagination : 200-203

ISBN : 978-2-7132-2138-5

ISSN : 0008-0055

Référence électronique

Odile Goerg, "Salm, Steven J. \& Falola, Toyin (eds.). - African Urban Spaces in Historical Perspective », Cahiers d'études africaines [En ligne], 185|2007, mis en ligne le 29 mars 2007, consulté le 24

septembre 2020. URL : http://journals.openedition.org/etudesafricaines/6858 ; DOI : https://doi.org/ 10.4000/etudesafricaines.6858

Ce document a été généré automatiquement le 24 septembre 2020.

(c) Cahiers d'Études africaines 


\section{Salm, Steven J. \& Falola, Toyin (eds.). - African Urban Spaces in Historical Perspective}

Rochester-Woodbridge, University of Rochester Press, 2005, XL+397 p., index.

Odile Goerg

1 Cet ouvrage reprend des communications présentées initialement lors d'un colloque portant sur les villes en Afrique, intitulé « African Urban Spaces. History and Culture ", qui avait rassemblé 140 contributions à Austin (Université du Texas) en 2003. La longue introduction (xv-XxxIx) rédigée par Catherine Coquery-Vidrovitch propose un vaste bilan des études urbaines, qui dépasse les articles publiés dans le volume et permet de les replacer dans le contexte historiographique global (périodisation, terminologie, évolution thématique). L'histoire urbaine connaît en effet un profond renouvellement depuis une vingtaine d'années et cet ouvrage entend contribuer à l'ouverture de nouvelles pistes.

2 Même restreinte à quinze contributions, la palette couverte par cet ouvrage reste impressionnante par la diversité thématique, la distribution géographique large ( 5 sur l'Afrique de l'Ouest, 4 sur l'Afrique australe, 3 sur l'Afrique orientale, 2 sur l'Afrique centrale), la variété disciplinaire ainsi que la durée concernée, allant de la période antérieure à la colonisation au très contemporain. Les articles sont organisés en quatre rubriques: "Constructing Built Space» (3), «Racialized and Divided Space» (4), «Shifting Space and Transforming Identities» (4), «Colonial Legacies and Devitalized Space » (4). Les critères de sélection ne sont pas explicités par les éditeurs du volume. De l'aveu même de ceux-ci (voir préface), l'organisation proposée a un côté arbitraire, parfois peu convaincant: certains articles pourraient se trouver tout autant sous d'autres rubriques ou répondent en fait à d'autres problématiques. Comme l'indique le titre, la volonté éditoriale était de mettre l'accent sur les espaces urbains; ceci est trompeur car les thématiques internes prennent souvent d'autres approches, que la notion d'espace est poly-sémantique, allant du très concret au plus abstrait. C. Coquery- 
Vidrovitch, dotée d'une très bonne connaissance de l'historiographie à la fois francophone et anglophone, met d'ailleurs en évidence la différence d'approche de l'espace dans les deux milieux de recherche, l'une plus géographique et spatiale (s'intéressant notamment aux aspects fonciers), l'autre plus abstraite (influencée notamment par les cultural ou post-modern studies). Cela aboutit en fait à une dilution de la catégorie " espace ", peu explicitée en général.

Ce n'est pas le cas de la contribution qui ouvre le livre. M. D. DeLancey pose en effet directement la question de l'espace, en analysant les critères d'organisation, selon l'orientation cardinale ou selon la centralité des bâtiments. À travers des exemples tirés de l'Empire de Sokoto et du Cameroun, il interroge la façon dont un héritage culturel et des changements postérieurs (nomadisme/sédentarisation, islamisation) peuvent être traduits spatialement et servir de facteurs de médiation interculturelle entre Peul, Hausa et Kanuri. Il souligne ainsi la position centrale successive du palais puis de la mosquée comme marque d'adhésion à un modèle religieux. Le raisonnement ouvre des pistes intéressantes, auxquelles on aurait pu ajouter le jeu sur le haut et le bas. S'il mentionne bien les recherches de Labelle Prussin, on peut s'étonner toutefois dans cette perspective de l'absence de référence au travail de Dominique Malaquais: "Constructing Power: Architecture, Landscape and the Design of Space in a Chieftaincy of the Bamileke Highlands ».

4 Comme souvent, dans un ouvrage collectif, l'intérêt et l'originalité des travaux varient beaucoup; cette impression est accentuée également par la diversité des approches disciplinaires : l'histoire domine avec 9 articles mais l'urbanisme et la géographie (4), l'anthropologie (1) et la littérature (1) sont aussi présents. Les attentes des lecteurs, variables selon leur propre formation et connaissances, doivent donc s'adapter à la perspective adoptée. Certains articles semblent plutôt des compilations sans grand apport novateur, sur des thèmes déjà bien cernés, tandis que d'autres présentent réellement une recherche originale. Ainsi, des géographes s'essaient à l'esquisse historique de longue durée, en gommant ce que pourrait être leur apport spécifique. Au-delà de la diversité, plusieurs thèmes transversaux apparaissent dont l'index facilite le repérage. Ainsi, on peut noter l'importance de l'analyse des discours et des représentations. Cette approche ne renouvelle cependant guère les questions, notamment celle des politiques ségrégatives dont les fondements ont déjà été largement étudiés. Est abordé aussi le lien entre l'organisation de l'espace, la définition de la ville et la perception des différences spatiales, la question de la projection de cultures sur des espaces ou celle des villes en relation avec des formes d'identité africaines ou nationales.

5 Se situant dans le courant des recherches actuelles, certains articles enjambent les périodes historiques et ignorent la rupture politique théoriquement marquée par les indépendances pour mettre l'accent au contraire sur les continuités et s'intéresser aux mutations induites. Des analyses portent par exemple sur les changements des politiques urbaines quand les ressources financières remplacent la "race » comme critère d'organisation de l'espace. L'étude des programmes d'habitat dans la petite ville d'Opuwo en Namibie (F. Müller-Friedman " "Just Build it Modern". Post-Apartheid Spaces on Namibia's Urban Frontier») montre par exemple la continuité de la perception de la ville au-delà de l'abrogation de l'apartheid. La modernité, symbolisée ici par un type de lotissement et d'architecture préconisés dans les années 1950, a modelé la notion de ville. Cette approche est une contribution importante à des 
questions plus générales : comment penser la ville autrement qu'en suivant, souvent inconsciemment, le modèle hérité des planificateurs antérieurs? Qui sont les théoriciens et les acteurs de la ville considérée malgré tout comme nouvelle? Quel rôle peuvent jouer les populations dans les politiques adoptées? Ces questions sont appréhendées aussi par D. Ferremenga dans le cadre du Zimbabwe ; il présente une vue d'ensemble de l'urbanisation et de la planification et met en évidence le fait que les besoins des plus pauvres ne sont guère mieux pris en compte actuellement que par le passé. Toute action sur la ville subit les contradictions et les difficultés du politique en général (la corruption; le refus de mécontenter d'anciens combattants de l'indépendance, alliés principaux du parti unique). 0 . Eno s'intéresse également au très contemporain en montrant comment Mogadiscio a souffert de plein fouet de la guerre civile (1991-2003) tandis que T. Ekali présente à grands traits le processus de déclin de Victoria au Cameroun de 1858 à 1982, en fonction des données politiques et économiques. Dans ces cas, la notion d'espace urbain recouvre celle de la ville dans son entier, sans réflexion particulière.

D'autres articles envisagent la thématique des réseaux en relation avec la conception de la ville. Ainsi, E. Ross propose une réflexion sur des fondations urbaines liées à l'islam confrérique en Sénégambie, bien avant la colonisation («From Marabout Republics to Autonomous Rural Communities »). Même si la notion même de "ville ", juste effleurée ici dans ses caractéristiques, ne fait pas l'objet d'analyses toujours convaincantes, la mise en évidence de racines anciennes pour l'installation humaine ainsi que la notion d'hybridité des agglomérations qui en découlent sont, elles, stimulantes. Ces agglomérations, au sens large du terme, prennent souvent leur essor au $x x^{e}$ siècle, à l'instar de Tivavouane, Kaolack, Touba ou Djourbel. J. Genova recourt à la littérature pour montrer que l'imaginaire de la ville comme élément de l'identité des pays africains indépendants (l'africanité) est contrasté : certains auteurs prônent son rejet, dans la lignée, faudrait-il ajouter, des courants dominants de la pensée coloniale, qui considère les Africains comme intrinsèquement ruraux; d'autres affirment au contraire sa nécessité. Le corpus mobilisé, limité à trois romans connus, est toutefois bien maigre pour aboutir à une conclusion somme toute attendue, tant ce paradigme parcourt toute l'histoire, non seulement de l'Afrique mais aussi d'autres zones en vif développement urbain.

7 La réflexion sur les identités, qui irrigue la recherche contemporaine, est présente à travers diverses contributions: l'étude des processus migratoires à Libreville, qu'il s'agisse de mouvements forcés ou volontaires, de travailleurs indochinois ou d'OuestAfricains attirés par les opportunités économiques, renforce l'idée de la ville comme creuset démographique et culturel (J. Rich). C. Sandwith analyse dans le cadre de Cape Town des années 1920 la concurrence entre deux groupes de l'élite et deux modèles éducatifs contrastés, l'un réformiste, l'autre plus radical. Le mode de vie adopté (habillement, éducation, habitat) reflète les prises de position. Dans les deux cas, l'éducation est conçue comme un moyen de changer la situation personnelle et politique, soit en conformité avec les valeurs prônées et véhiculées par la colonisation, soit en préconisant un changement révolutionnaire. Autant que d'histoire urbaine, il s'agit en fait d'histoire sociale et politique montrant les choix effectués par des membres de la bourgeoisie africaine dans le contexte de la ségrégation. W. Visser s'intéresse à l'autre bout de l'échelle sociale. En s'intéressant aux ouvriers afrikaners des villes minières, il cherche à mettre en évidence comment ceux-ci développèrent un aspect de l'identité afrikaner propre à leur groupe, identité qui diffère en partie du 
nationalisme afrikaner élaboré par les élites politiques au même moment. Il pointe notamment des particularités culturelles, comme les courses de chien ou le sens particulier donné aux commémorations du Grand Trek, comme moments de fierté pour ce prolétariat blanc.

8 La question de la sécurité, cruciale dans bien des villes actuellement, commence à attirer désormais certains chercheurs. Ceux-ci démontrent les racines anciennes du contrôle de la criminalité comme forme de gestion de l'espace. Dans son étude comparative entre Lagos et Ibadan, L. Fourchard se concentre sur l'Entre-deux-guerres pour analyser le développement de la délinquance et les moyens de lutte mis en œuvre contre le crime, moyens aussi bien publics que privés, en relation avec l'accroissement de la misère urbaine. Il faut espérer que cette contribution novatrice, basée notamment sur les archives de la police, suscitera d'autres études sur ce thème encore peu exploré.

$\mathrm{Au}$ final, ce livre s'avère stimulant parfois, banal dans d'autres cas tant on a l'impression, dans certaines contributions, d'une méconnaissance des recherches déjà effectuées, notamment dans le domaine francophone, ou d'assister à des recherches en cours. Il est vrai que l'histoire urbaine de l'Afrique a pris de telles proportions actuellement, que sa maîtrise globale est de moins en moins possible et qu'il faut donc creuser ses propres nouveaux sillons. On peut saluer dans cette perspective l'accent mis sur des villes moyennes, moins connues que les grandes métropoles. C'est le cas, entre autres, d'Opuwo déjà cité ou de l'étude d'Isiolo au Kenya. M. N. Amutabi analyse les facteurs externes à l'œuvre dans le développement de ce centre (exploitation de rivalités entres groupes; favoritisme des Chrétiens face aux Musulmans; rapports entre éleveurs et agriculteurs...) ainsi que la marge de manœuvre des habitants, leur agency, dans ce contexte.

$\mathrm{Au}$ niveau de la forme et de l'agencement, on peut regretter que l'ouvrage ne comprenne pas de bibliographie générale alors que certains articles sont dotés d'une liste de références, d'autres non. 